\title{
Attendance at an outpatient follow-up clinic by HIV-positive psychiatric patients initiated on ART as inpatients
}

\author{
Y M Nel, MMed (Psych), FCPsych (SA); G Jonsson, MMed (Psych) FCPsych (SA) \\ Department of Psychiatry, Faculty of Health Sciences, University of the Witwatersrand, Johannesburg, South Africa
}

Corresponding author: Y Nel (yvette.nel@wits.ac.za)

\begin{abstract}
Background. Evidence suggests that the presence of mental illness may be associated with poorer adherence to antiretroviral therapy (ART). There is also a general understanding that patients initiated on ART as inpatients have poorer outcomes than those initiated as outpatients. Negative perceptions regarding future adherence may affect the clinical decision to initiate ART in hospitalised psychiatric patients. Attendance at clinic appointments is an indicator of medication adherence, and is easily measurable in a limited-resource setting. Objectives. The primary objective of this study was to examine the rate of attendance at the first clinic appointment post discharge from a period of psychiatric hospitalisation in HIV-positive psychiatric patients initiated on ART as inpatients. A secondary objective was to determine which factors, if any, were associated with clinic attendance.

Methods. This study was a retrospective record review, conducted at the Luthando Neuropsychiatric HIV Clinic in Soweto, which is an integrated mental healthcare and ART clinic. Patients who were initiated on ART as psychiatric inpatients from 1 July 2009 to 31 December 2010, and subsequently discharged for outpatient follow-up at Luthando Clinic were included in the sample.

Results. There were 98 patients included in the analysis. The sample was predominantly female. The rate of attendance was $80 \%$. The attendant and non-attendant groups were similar in terms of demographic and clinical data. Significantly fewer non-attendant patients had disclosed their HIV status to their treatment supporter $(p=0.01)$.

Conclusion. Non-disclosure of HIV status needs to be further addressed in integrated psychiatric HIV treatment facilities in order to improve attendance. Female predominance in this setting should also be further investigated.
\end{abstract}

S Afr J Psychiatr 2015;21(3):98-102. DOI:10.7196/SAJP.8254

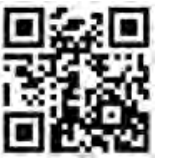

The interaction between HIV infection and mental illness is complex. HIV has a causative role in mental illness, and the mentally ill may be comorbidly infected with HIV, with some authors suggesting an increased risk of infection in the mentally ill. ${ }^{[1]}$ Adherence to antiretroviral treatment (ART) is vital for virological suppression and successful long-term management. ${ }^{[2]}$ There is some evidence to suggest that mental illness is associated with poorer adherence to ART regimens, with reports that neurocognitive disorders and serious mental illness may be negatively associated with adherence. ${ }^{[3,4]}$ Research has also shown that medical patients initiated on ART as inpatients have poorer outcomes than those initiated as outpatients. ${ }^{[5,6]}$ Negative perceptions with regard to rates of adherence, future clinic attendance and long-term outcome may affect the decision to initiate ART in HIV-positive psychiatric patients, especially in patients requiring hospitalisation, in effect depriving these patients of potentially lifesaving treatment. ${ }^{[7]}$

With this in mind, the Luthando Neuropsychiatric HIV Clinic (Luthando Clinic) at Chris Hani Baragwanath Academic Hospital (CHBAH), Soweto, was established, providing specialised integrated treatment for HIV-positive psychiatric patients at a tertiary academic level. The Luthando Clinic offers inpatient and outpatient services for all HIV-positive patients at CHBAH with a psychiatric diagnosis. Patients are thus able to attend one clinic for both their psychiatric treatment and their ART. In addition, patients have access to a full multidisciplinary team, including occupational therapists, psychologists and social workers. They are also afforded the opportunity to attend support groups. While it is common practice to delay initiation of ART until a patient is discharged from the medical wards at $\mathrm{CHBAH}$, hospitalised psychiatric patients are initiated on ART at the Luthando Clinic if urgent ART treatment is clinically indicated, e.g. when a diagnosis of psychosis secondary to HIV infection is suspected. Patients initiated on ART while in the psychiatric ward are subsequently discharged with an appointment date to follow up at the Luthando Clinic within a month of discharge.

Measures of adherence in the research setting include self-report, electronic monitoring caps, pill counts, biological markers, drug levels, as well as regular clinic attendance. ${ }^{[8]}$ Reasons why patients may fail to attend clinic appointments include patient choice, forgetfulness, acute illness, hospitalisation, transfer to another treatment site and death. ${ }^{[9]}$ Research has shown an association between clinic attendance and adherence to medication. ${ }^{[10]}$ Integrated HIV and mental healthcare might result in improved rates of clinic attendance, by providing personalised follow-up, specifically tailored to the needs of those with a mental illness as well as HIV infection. ${ }^{[11]}$ In a previous study conducted at the Luthando clinic, Wagner et al. ${ }^{[12]}$ suggest that rates of retention in care at the Luthando Clinic are similar to those reported for general HIV clinics, and higher than expected for psychiatric 
clinics, with $24.8 \%$ of patients lost to followup (LTFU), defining LTFU as failure to attend the clinic for more than 6 months.

Attendance at the first clinic appointment post discharge from hospitalisation is the first step towards long-term retention in care and positive overall outcome. First clinic appointment attendance post discharge from hospitalisation has previously been studied internationally in the psychiatric setting, with non-attendance ranging from $18 \%$ to $64 \% .^{[13,14]}$ To our knowledge, this has not been directly studied in an integrated HIV and mental healthcare centre, or in a South African (SA) setting. Factors found to be associated with clinic attendance at ART clinics and at mental health clinics have generally been inconsistent but include a variety of sociodemographic and clinical factors. ${ }^{[13-16]}$ Patients LTFU at the Luthando Clinic in a previous study cited logistical and financial reasons for clinic non-attendance, as well as miscommunication concerning the follow-up appointment date. ${ }^{[12]}$

A larger evidence base regarding the prescription of ART in the mentally ill, subsequent follow-up, adherence to ART regimens and long-term retention in care after inpatient initiation of ART in the mentally ill will hopefully help guide future research and tailor treatment guidelines to better serve this potentially vulnerable population.

\section{Objectives}

The primary objective of this study was to examine the rate of attendance at the first outpatient clinic appointment post discharge from psychiatric hospitalisation in HIV-positive psychiatric patients initiated on ART as inpatients. Secondary objectives were to describe the demographic and clinical profile of inpatients initiated on ART, to compare attendant and non-attendant groups with regard to sociodemographic and clinical data, and to determine which factors, if any, might be associated with clinic attendance.

\section{Method}

\section{Selection of study participants}

The study was a retrospective record review conducted at the CHBAH Luthando Clinic. The final sample consisted of 98 HIV-positive, ART-naive patients who were initiated on ART between 1 July 2009 and 31 December

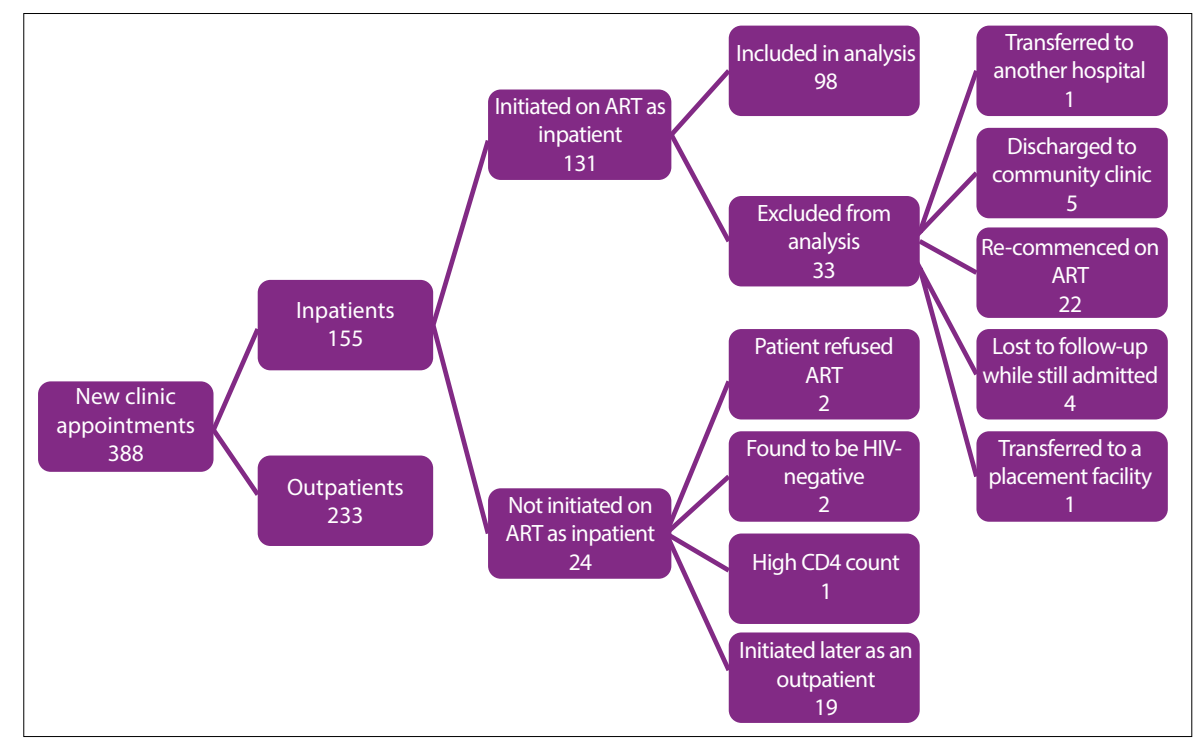

Fig. 1. Flow chart outlining all new patient clinic appointments at the Luthando Neuropsychiatric Clinic for the period 1 July 2009 - 31 December 2010.

2010. The total number of new patients attending the Luthando Clinic in the study period was $388 ; 155$ of these new patients were inpatients at the time of referral (Fig. 1). Files were included in the analysis if the patient had been admitted to a psychiatric ward in this period and initiated on ART as an inpatient, and then discharged to follow-up at the Luthando Clinic with an appointment date. Patients were excluded if ART was initiated while they were outpatients, if they had been transferred to another hospital or placement facility after inpatient initiation with no follow-up appointment, if they were discharged to follow-up at another community clinic, if they were LTFU while still admitted or if they had previously defaulted ART. LTFU while still admitted occurred in four medically unwell patients who were transferred to medical wards for further management and subsequently managed by the medical department of the hospital (Fig. 1). The study was approved by the University of the Witwatersrand's Human Research Ethics Committee.

\section{Data collection}

Data were retrospectively recorded from the clinic or hospital file of each patient included in the study. Patients were regarded as attendant if they attended the clinic on the appointment date or within 1 month of the appointment date, and non-attendant if they did not attend the clinic within 1 month of appointment date.

\section{Statistics}

Statistical analysis was performed using the GraphPad Instat3 program. The association between clinic attendance/non-attendance and categorical variables was analysed using Fisher's exact test. The association between clinic attendance/non-attendance and continuous variables was analysed using the unpaired $t$-test, or the non-parametric MannWhitney test for non-normally distributed data.

\section{Results \\ Rate of attendance}

Of the final sample of 98 patients, the rate of attendance was $80 \%(n=78)$, with $53 \%$ attending on the given appointment date and $27 \%$ attending within 1 month of the given appointment.

\section{Description of the sample}

The majority of the patients in the total sample were female (77\%) $(n=75)$. The mean age (standard deviation (SD)) of the sample was 34.9 (8.9) years (Table 1). Most of the sample were unemployed, accounting for $89 \%(n=87)$ of the sample. However, only $23 \%(n=23)$ of the sample were on a disability grant. The majority $(66 \%)(n=65)$ of the sample were single. The median (interquartile range (IQR)) number of years of schooling was $10(9-12)$ years. Of the total sample, 58\% lived within $10 \mathrm{~km}$ of the clinic. A history of substance use was recorded in $23 \%$ of the overall sample. In $10 \%$ of files reviewed, a 
Table 1. Comparison of demographic and clinical data of attendant v. non-attendant groups

\begin{tabular}{|c|c|c|c|c|}
\hline Data & Total $(N=98)$ & Attendant $(n=78)$ & Non-attendant $(n=20)$ & $p$-value \\
\hline Age (years), mean (SD) & $34.9(8.9)$ & $35.7(9.0)$ & $31.7(7.7)$ & $0.08^{*}$ \\
\hline Female, $n(\%)$ & $75(77)$ & $61(78)$ & $14(70)$ & 0.55 \\
\hline Unemployed, $n(\%)$ & $87(89)$ & $69(88)$ & $18(90)$ & 1.00 \\
\hline Disability grant $(n=97), n(\%)$ & $23(24)$ & $17(22)$ & $6(30)$ & 0.56 \\
\hline School education (years) $(n=79)$, median (IQR) & $10(9-12)$ & $10(9-11)$ & $11(10-12)$ & $0.08^{*}$ \\
\hline Substance use history $(n=88), n(\%)$ & $23(26)$ & $16(22)$ & $7(44)$ & 0.11 \\
\hline HIV status not disclosed $(n=51), n(\%)$ & $12(24)$ & $6(15)$ & $6(55)$ & $0.01^{*}$ \\
\hline WHO stage four $(n=88), n(\%)$ & $62(71)$ & $49(71)$ & $13(68)$ & 1.00 \\
\hline Nadir CD4 (cells $\left./ \mathrm{mm}^{3}\right)$, mean (SD) & $151.4(115.3)$ & $148.4(114.6)$ & $163.3(120.1)$ & $0.74^{*}$ \\
\hline Current partner, $n(\%)$ & $28(29)$ & $26(33)$ & $2(10)$ & 0.05 \\
\hline Length of hospitalisation (days), median (IQR) & $35.5(24.0-51.3)$ & $32.0(25.8-51.3)$ & $37.5(18.8-52.8)$ & $0.42^{\dagger}$ \\
\hline Distance from the clinic $<10 \mathrm{~km}(n=76), n(\%)$ & $44(58)$ & $34(55)$ & $10(71)$ & 0.37 \\
\hline $\begin{array}{l}p \text {-values calculated using Fisher's exact test unless otherwise stated. } \\
\text { *Unpaired } t \text {-test. } \\
{ }^{*} \text { Mann-Whitney test. } \\
\text { " Significant. }\end{array}$ & & & & \\
\hline
\end{tabular}

substance use history had not been enquired about. In $48 \%$ of the sample, it was not stated clearly if the diagnosis had been disclosed to the treatment supporter. The mean (SD) nadir CD4 count was 151.4 (115.3) (Table 1).

Data on psychiatric diagnosis were complicated by the lack of a definitive single diagnosis in $51 \%$ of the sample. Only $49 \%$ of the sample was labelled with one clear diagnosis. The most common differential diagnosis overall was psychotic disorder due to HIV (68 patients), followed by mood disorder due to HIV (31 patients), followed by bipolar disorder (not specified I or II) (20 patients) (Table 2). The International HIV Dementia Scale (IHDS) score was missing in $78 \%$ of the total sample and was therefore excluded from further analysis.

\section{Comparison of data: Attendant v. non- attendant groups}

The attendant and non-attendant groups were similar in terms of demographic and clinical data (Table 1$)$. The numbers $(n)$ are stated in Table 1 and where they differ from the total, the difference can be accounted for by missing data. There were no significant differences between attendant and nonattendant groups in terms of gender, age, employment status, disability-grant status, level of education, distance from the clinic or marital status. Similarly, there were no significant differences between the groups in terms of clinical data including World Health

Table 2. Outline of attendant $v$. non-attendant groups by psychiatric diagnosis

\begin{tabular}{llll}
\hline Diagnosis & $\begin{array}{l}\text { Total }(\mathbf{N}=\mathbf{9 8}), \\
\boldsymbol{n}(\%)\end{array}$ & $\begin{array}{l}\text { Attendant } \\
(\boldsymbol{n}=\mathbf{7 8}), \boldsymbol{n}(\%)\end{array}$ & $\begin{array}{l}\text { Non-attendant } \\
(\boldsymbol{n}=\mathbf{2 0}), \boldsymbol{n}(\mathbf{\%})\end{array}$ \\
\hline Primary psychiatric diagnosis & $47(48)$ & $34(44)$ & $13(65)$ \\
Bipolar disorder & $20(20)$ & $15(19)$ & $5(25)$ \\
Substance-induced psychotic disorder & $11(12)$ & $7(9)$ & $4(20)$ \\
Major depressive disorder & $9(9)$ & $8(10)$ & $1(5)$ \\
Schizophrenia & $8(8)$ & $5(6)$ & $3(15)$ \\
Schizoaffective disorder & $1(1)$ & $0(0)$ & $1(5)$ \\
Psychiatric diagnosis due to HIV/medical illness & $69(70)$ & $58(74)$ & $11(55)$ \\
Psychotic disorder due to GMC-HIV & $68(69)$ & $55(71)$ & $13(65)$ \\
Mood disorder due to GMC-HIV & $31(32)$ & $26(33)$ & $5(25)$ \\
HIV-associated neurocognitive disorder & $7(7)$ & $5(6)$ & $2(10)$ \\
Psychotic disorder due to GMC-other & $7(7)$ & $6(8)$ & $1(5)$ \\
GMC = general medical condition. & & &
\end{tabular}

Organization (WHO) stage of HIV infection, nadir CD4 count, length of hospitalisation, time to follow-up appointment, prescription of monotherapy v. polytherapy and substance abuse history. When data were grouped for analysis (because of small sample size), there was no significant association between attendance/non-attendance and the presence or absence of a 'primary psychiatric diagnosis' (Fisher's exact test, $p=0.13$ ) or the presence or absence of a 'psychiatric diagnosis due to general medical condition (HIV or medical illness)' (Fisher's exact test, $p=0.11$ ) (Table 2). The only statistically significant difference between the attendant and non-attendant groups was disclosure of HIV status. Only $15 \%$ of the non-attendant group had disclosed their HIV-positive status to the identified treatment supporter compared with $55 \%$ of the attendant group (Fisher's exact test, $p=0.01$ ) (Table 1). However, this information was missing in $48 \%$ of files reviewed, and should therefore be interpreted with caution.

\section{Discussion}

The reason for non-attendance (20\%) was not ascertained in this study, and most likely included patients who had discontinued ART treatment, were too unwell to return 
for follow-up appointments, or had moved to a different area and therefore changed clinics, as well as those that died during the time from discharge to follow-up appointment. ${ }^{[6,9]}$ To our knowledge, there is no other literature examining early attendance rates at an integrated neuropsychiatric clinic in SA following inpatient initiation of ART. Integrated care has been shown to improve outcomes, with a number of different models of mental health and HIV integrated programmes described in the literature. ${ }^{[11]}$ The rate of attendance found at our integrated clinic is comparable with rates of first clinic appointment attendance post discharge from psychiatric hospitalisation internationally. ${ }^{[13,14]}$ There are no data with which to compare our rate of attendance with early attendance post hospitalisation at HIV treatment centres in order to evaluate the effectiveness of the integrated model in terms of improving outcomes.

With regard to the impact of inpatient initiation of ART on subsequent clinic attendance, the literature suggests higher rates of LTFU and death post inpatient initiation of ART in medical wards than with outpatient initiation. ${ }^{[5,6]}$ It has previously been suggested that decreased time before initiation of treatment, resulting in a decreased number of preparation sessions might in part account for this finding. ${ }^{[5]}$ Time from diagnosis to initiation of ART was not examined in the current study. There may be links between illness awareness, insight into risk of infection, insight into need for treatment and subsequent adherence to treatment. ${ }^{[17]}$ This may have influenced the rate of attendance in the current study as all the patients presented primarily with a psychiatric diagnosis and not for HIV testing as a result of feared exposure, suggesting the primary focus was not on HIV testing and need for ART treatment, even though the mean CD4 count suggested that the majority of the sample required ART according to national guidelines at the time.

This predominantly female sample differs from a previous published description of psychiatric inpatients at CHBAH from 1996, with a distinct male predominance, females representing only $36 \%$ of the sample. ${ }^{[18]}$ It is unclear what the male:female ratio of acute psychiatric admissions is currently at CHBAH. The mid-year SA national statistics for $2011^{[19]}$ estimated a female:male HIV prevalence ratio of 1.5:1 in South Africans between the ages of 15 and 49 years. Studies at ART centres within SA have found a female predominance ranging from $62.8 \%$ to $71 \% .{ }^{[20,21]}$ The increased prevalence of HIV infection in females in the SA context is attributable to biological factors, related to the female anatomy, as well as psychosocial factors, including gender-based violence and transactional sex, which have been shown to impact rates of transmission and vulnerability to HIV infection. ${ }^{[22]}$ It has also been suggested that there is an increase in risk for psychiatric conditions in females with HIV, compared with males. ${ }^{[23]}$ In addition, fewer men in Soweto than women have tested for HIV. ${ }^{[24]}$ It is possible that fewer men in the psychiatric wards are referred for, or agree to, HIV testing at CHBAH.

Clinically, most patients presented with a psychotic disorder diagnosis. Screening for HIV-associated neurocognitive disorder (HAND) may not have been adequate in the sample, possibly as a result of the psychotic presentation of most patients, thus underestimating the prevalence of HAND. A report on psychiatric admissions to CHBAH in 1996, before the national roll-out of ART, suggested the most common diagnoses in their sample were schizophrenia and bipolar disorder. ${ }^{[18]}$ It should be noted that in 1996, 'psychotic disorder due to HIV' was an emerging concept and was not listed as a diagnosis in their sample. In the current study, a substance use history was not obtained at all in $10.2 \%$ of patients' records. This may suggest that clinicians underestimate the need for substance use screening in the HIV-positive psychiatric patient, therefore potentially underdiagnosing substance use disorders. Previously it has been shown that substance abuse is associated with poorer adherence to ART, and that integrating HIV, mental health and substance abuse care may lead to further improved outcomes. ${ }^{[8,11]}$

Although a number of factors were compared between the attendant and non-attendant groups, the only significant difference between the groups was disclosure of HIV status, with non-attendance being associated with non-disclosure of HIV status. The lack of significance of other factors previously found to be important in attendance may have been influenced by the small final sample size. It was a requirement prior to initiation of ART that patients had a treatment supporter. The treatment supporter was not always aware of the diagnosis at the time of initiation of ART. It has been previously recommended that patients should have disclosed to one significant other or joined a support group prior to initiation of ART. ${ }^{[25]}$ Patients are actively encouraged to attend Luthando support groups prior to initiation of ART. Consequently disclosure was not always insisted on by the treating team. It is not known why additional information detailing specific disclosure of HIV status was only recorded in $52 \%$ of the files reviewed, and the large amount of missing data for this variable should be taken into account when assessing the significance of this finding. However, the finding in this study that disclosure of HIV status is significantly associated with clinic attendance is consistent with the findings in previous African studies examining adherence to ART. ${ }^{[26]}$ Reasons for non-disclosure were not examined in this study, but have previously been extensively studied. Stigma related to disclosure of HIV status has been identified as a significant barrier to adherence. ${ }^{[25]}$ Furthermore, gender-based violence and subsequent disempowerment of women may influence behaviour related to disclosure of HIV status among women in Soweto, which might be significant, given the female predominance in this study. ${ }^{[22,27]}$ The patients in the current study have the dual stigma associated with HIV infection as well as a psychiatric diagnosis. Stigma has been reported extensively in psychiatric populations. ${ }^{[28]}$ Stigma regarding psychiatric illness may influence an individual's decision to disclose a psychiatric diagnosis. It seems from previous research that disclosure of HIV status, if not met by rejection, serves the purpose of providing the patient with emotional support, as well as someone to remind the patient to attend the clinic. ${ }^{[25]}$ It is therefore important that non-disclosure of HIV status proved to be the only significant association with non-attendance at the clinic.

It was hypothesised that there would be an association between psychiatric diagnosis and attendance. However, it was not possible to demonstrate any associations with individual psychiatric conditions and adherence in the current study because of the small numbers diagnosed with each psychiatric condition, as well as the lack of definitive diagnosis in many of the records. Overall there was a high number of patients with a psychotic presentation with relatively fewer patients overall diagnosed with a major depressive episode. This was most likely a result of the selection of hospitalised psychiatric patients for the study. 


\section{Study limitations}

Although 388 files were reviewed, only 98 were relevant for the current study. This figure was smaller than expected, and the percentage of non-attendant patients was even smaller, which impacted on the power of the study. Owing to the retrospective nature of this study, completeness of data was dependent on physician record-keeping. Several variables were poorly recorded in the Luthando Clinic, $\mathrm{CHBAH}$ and outpatient psychiatric files. The most noticeable of these was the IHDS score. There were no objective tools used to make psychiatric diagnosis, or to diagnose substance abuse, and the data were purely recorded from clinician's notes. Most patients had no definitive diagnosis, which made it difficult to compare groups based on psychiatric diagnosis. In terms of substance use, self-report may not be reliable, especially if current use may influence the clinician's decision to initiate ART and the patient is aware of this. ${ }^{[8]}$ The absence of an outpatient ART initiation control group is a limitation of this study with regard to quantifying the significance of inpatient initiation of ART.

\section{Conclusion}

This study provides vital information with regard to the demographic profile of psychiatric inpatients initiated on ART, most notably highlighting a female predominance. It also reinforces the potential significance of disclosure of HIV status. The study brings to attention system difficulties, reinforcing the need for meticulous recordkeeping. Potential targets for improvement in daily operations at the clinic include encouragement of disclosure of HIV status aimed at improving clinic attendance.

Acknowledgements. This study forms part of a research report previously submitted to the University of the Witwatersrand by YN for an MMed degree, supervised by GJ. Thank you to Dr P Gaylard for assistance with statistical review.

\section{References}

1. Carey MP, Carey KB, Kalichman SC. Risk for human immunodeficiency virus (HIV) infection among persons with severe mental illness. Clin Psychol Rev 1997;17(3):271-291. [http:// dx.doi.org/10.1016/s0272-7358(97)00019-6]

2. Richard N, Juntilla M, Abraha A, et al. High prevalence of antiretroviral resistance in treated Ugandans infected with non-subtype B human immunodeficiency virus type 1. AIDS Res Hum Retroviruses 2004;20(4):355-364. [http://dx.doi.org/10.1089/088922204323048104]

3. Ettenhofer ML, Foley J, Castellon S, Hinkin C. Reciprocal prediction of medication adherence and neurocognition in HIV/AIDS. Neurology 2010;74(15):1217-1222. [http://dx.doi. org/10.1212/wnl.0b013e3181d8clca]

4. Moore D, Posada C, Parikh M, et al. HIV-infected individuals with co-occurring bipolar disorder evidence poor antiretroviral and psychiatric medication adherence. AIDS Behav 2012;16(8):2257-2266. [http://dx.doi.org/10.1007/s10461-011-0072-2]

5. Boyles T, Wilkinson L, Leisegang R, Maartens G. Factors influencing retention in care after starting antiretroviral therapy in a rural South African programme. PLoS One 2011;6(5):e19201. [http://dx.doi.org/10.1371/journal.pone.0019201]

6. Eshun-Wilson I, van der Plas H, Prozesky HW, Zeier MD, Taljaard JJ, Nachega J. Combined antiretroviral treatment initiation during hospitalization: Outcomes in South African adults. I Acquir Immune Defic Syndr 2009;51(1):105-106. [http://dx.doi.org/10.1097/ qai.0b013e3181963cd4]

7. Bangsberg DR, Moss A. When should we delay highly active antiretroviral therapy? J Gen Intern Med 1999;14(7):446-448. [http://dx.doi.org/10.1046/j.1525-1497.1999.05109.x]

8. Uldall KK, Palmer NB, Whetten KK, Mellins C. Adherence in people living with HIV/AIDS, mental illness, and chemical dependency: A review of the literature. AIDS Care 2004;16(suppl 1):s71-s96. [http://dx.doi.org/10.1080/09540120412331315277]

9. Geng H, Bangsberg R, Musinguzi N, et al. Understanding reasons for and outcomes of patients lost to follow-up in antiretroviral therapy programs in Africa through a sampling-based approach. J Acquir Immune Defic Syndr 2010;53(3):405-411. [http://dx.doi.org/10.1097/ qai.0b013e3181b843f0]

10. Kunutsor S, Walley J, Katabira E, et al. Clinic attendance for medication refills and medication adherence amongst an antiretroviral treatment cohort in Uganda: A prospective study. AIDS Res Treat 2010; 2010:872396(Epub):1-7. [http://dx.doi.org/10.1155/2010/872396]

11. Soto TA, Bell J, Pillen M. Literature on integrated HIV care: A review. AIDS Care 2004;16(suppl 1):s43-s55.

12. Wagner T, Furin J, Gripshover B, Jeenah Y, Jonsson G. Loss to follow-up among a group of patients with HIV and severe mental illness in South Africa. World J AIDS 2014;4:74-80. [http://dx.doi.org/10.4236/wja.2014.41009]

13. Crompton MT, Rudisch E, Craw J, Thompson T, Owens DA. Predictors of missed first appointments at community mental health centres after psychiatric hospitalization. Psychiatr Serv 2006;57(4):531-537. [http://dx.doi.org/10.1176/appi.ps.57.4.531]

14. Kruse G, Rohland M. Factors associated with attendance at a first appointment after discharge from a psychiatric hospital. Psychiatr Serv 2002;53(4):473-476. [http://dx.doi.org/10.1176/ appi.ps.53.4.473]

15. Catz SL, McClure JB, Jones GN, Brantley PJ. Predictors of outpatient medical appointment attendance among persons with HIV. AIDS Care 1999;11(3):361-373. [http://dx.doi. org/10.1080/09540129947983]

16. Killaspy H, Banerjee S, King M, Lloyd M. Prospective controlled study of psychiatric outpatient non-attendance: Characteristics and outcome. Br J Psychiatry 2000;176:160-165. [http://dx.doi.org/10.1192/bjp.176.2.160]

17. Maqutu D, Zewotir T, North D, Naidoo K, Grobler A. Determinants of optimal adherence over time to therapy amongst HIV positive adults in South Africa: A longitudinal study. AIDS Behav 2011;15(7):1465-1474. [http://dx.doi.org/10.1007/s10461-010-9688-x]

18. Behr GM, Christie C, Soderlund N, Lee T. Patterns and determinants of acute psychiatric readmissions. S Afr Med J 2002;92(5):369-374.

19. Statistics South Africa. www.statssa.gov.za. Mid-year population estimates 2011. beta2.statssa. gov.za/publications/P0302/P03022011.pdf (accessed 17 February 2014).

20. Brennan AT, Maskew M, Fox MP. The importance of clinic attendance in the first six months on antiretroviral treatment: A retrospective analysis at a large public sector clinic in South Africa. J Int AIDS Soc 2010;13(49). [http://dx.doi.org/10.1186/1758-2652-13-49]

21. Nachega JB, Stein DM, Lehman DA, et al. Adherence to antiretroviral therapy in HIV-infected adults in Soweto, South Africa. AIDS Res Hum Retroviruses 2004;20(10):1053-1056. [http:// dx.doi.org/10.1089/aid.2004.20.1053

22. Ackermann L, de Klerk GW. Social factors that make South African women vulnerable to HIV infection. Health Care Women Int 2002;23(2):163-172. [http://dx.doi org/10.1080/073993302753429031]

23. Brandt R. The mental health of people living with HIV/AIDS in Africa: A systemic review. Af J AIDS Res 2009;8(2):123-133. [http://dx.doi.org/10.2989/ajar.2009.8.2.1.853]

24. Mhlongo S, Dietrich J, Otwombe KN, Robertson G, Coates TJ, Gray G. Factors associated with not testing for HIV and consistent condom use among men in Soweto, South Africa. PLoS One 2013;8(5):e62637. [http://dx.doi.org/10.1371/journal.pone.0062637]

25. Nachega J, Knowlton AR, Deluca A, et al. Treatment supporter to improve adherence to antiretrovial therapy in HIV-infected South African adults. A qualitative study. J Acqui Immune Defic Syndr 2006;43(supplement 1):127-133. [http://dx.doi.org/10.1097/01. qai.0000248349.25630.3d]

26. Do NT, Phiri K, Bussman H, Gaolathe T, Marlink RG, Wester WC Psychosocial factors affecting medication adherence among HIV-1 infected adults receiving combination antiretroviral therapy (cART) in Botswana. AIDS Res Hum Retroviruses 2010;26(6):685-691. [http://dx.doi.org/10.1089/aid.2009.0222]

27. Dunkle K, Jewkes R, Brown H, McIntyre J, Gray G, Harlow S. Gender-based violence and HIV infection among pregnant women in Soweto. A technical report to the Australian agency for international development. Aus AID; 2003. Report No.: ISBN: 1-919809-78-3. www.mrc.ac.za/ gender/women.pdf (accessed 3 June 2015)

28. Gray AJ. Stigma in psychiatry. J Royal Soc Med 2002;95(2):72-76. [http://dx.doi.org/10.1258/ jrsm.95.2.72] 